Article

\title{
Optimal Coordinated Planning of Energy Storage and Tie-Lines to Boost Flexibility with High Wind Power Integration
}

\author{
Fahad Alismail 1,2,3 ${ }^{(D)}$, Mohamed A. Abdulgali1 1,*(D) and Muhammad Khalid 1,3 \\ 1 Electrical Engineering Department, King Fahd University of Petroleum \& Minerals, \\ Dhahran 31261, Saudi Arabia; fsalismail@kfupm.edu.sa (F.A.); mkhalid@kfupm.edu.sa (M.K.) \\ 2 Center for Environment \& Water, King Fahd University of Petroleum \& Minerals (KFUPM), \\ Dhahran 31261, Saudi Arabia \\ 3 K.A.CARE Energy Research \& Innovation Center, Dhahran 31261, Saudi Arabia \\ * Correspondence: atta@kfupm.edu.sa
}

check for

updates

Citation: Alismail, F.;

Abdulgalil, M.A.; Khalid, M. Optimal

Coordinated Planning of Energy

Storage and Tie-Lines to Boost

Flexibility with High Wind Power

Integration. Sustainability 2021, 13,

2526. https://doi.org/13052526

Academic Editor: Konstantin Volkov

Received: 13 January 2021

Accepted: 21 February 2021

Published: 26 February 2021

Publisher's Note: MDPI stays neutral with regard to jurisdictional clai$\mathrm{ms}$ in published maps and institutional affiliations.

Copyright: $\odot 2021$ by the authors. Licensee MDPI, Basel, Switzerland. This article is an open access article distributed under the terms and conditions of the Creative Commons Attribution (CC BY) license (https:// creativecommons.org/licenses/by/ $4.0 /)$.

\begin{abstract}
Since renewable power is intermittent and uncertain, modern grid systems need to be more elegant to provide a reliable, affordable, and sustainable power supply. This paper introduces a robust optimal planning strategy to find the location and the size of an energy storage system (ESS) and feeders. It aims to accommodate the wind power energy integration to serve the future demand growth under uncertainties. The methodology was tested in the IEEE RTS-96 system and the simulation results demonstrate the effectiveness of the proposed optimal sizing strategy. The findings validate the improvements in the power system reliability and flexibility.
\end{abstract}

Keywords: optimization technique; energy management system; reliability; energy storage system; power system planning; renewable energy

\section{Introduction}

Modern power system design approaches aim to cut output, maintenance, and utility costs, boost flexibility, minimize emissions, and increase energy efficiency [1]. Uncentralism means that due to the introduction of distributed generation systems, the flexibility of the grid has improved. Usually, several power systems are linked to each other and related to renewable energy sources which paves the way to the smart grid evolution. Such alternative resources may make the network more economical than centralized systems that rely on conventional plants for generation. As the options for renewable energy are integrated into the power grid, operating costs are subject to decrease. This is because the cost of operating renewable energy projects is minimal compared to conventional generators that rely on high prices of fuel. Renewable resources are readily available for generating electricity, but investment costs are needed to transform renewable energy into electricity. The authors in [2] emphasize the energy storage system (ESS) as a primary technology to be considered when integrating distributed renewable generation systems into the smart grid to reduce the intermittency of such tools. There is a number of storage systems that are rapidly emerging and being incorporated into the power grids [3]. In numerous studies ESS is introduced to solve various operating problems of power systems. One of the most important applications [4] is their contribution to provide emergency load assistance.

The ESS could make power systems operation more efficient by charging and storing electric power during low-cost cycles. In comparison, the energy stored during highpriced [5] cycles could be discharged and supplied to the grid. This process will also lead to a more sustainable operation, with reduced operating costs. The embodying of renewable energy sources and ESS increases the supply of electricity, which should be well regarded in the planning studies of any sustainable power generation systems [6]. A feasibility study of an ESS for insulated microgrids was conducted by the authors in [7] to show its benefits.

Before being incorporated into the power system, ESSs should be optimally sized by reducing the total cost feature, which includes ESS operating and investment costs $[4,8]$. 
Ref. [9] contains the costs and income for the mutual energy in the total expenditures for network-connected power systems. As the size of the ESS increases, cost of investment is increasing linearly. Operating costs therefore decrease exponentially as the scale of the ESS increases [5]. The intent is to determine the size of the ESS at the minimum overall cost [10]. After integrating the ESS to the system, the system will work in a more economical and environment-friendly way.

Several deterministic and probabilistic optimization methods were evaluated to solve the optimal sizing problem of the ESS, strategies include linear programming (LP) [11], mixed-integer linear programming (MILP) [4], mixed-integer non-linear programming (MINLP) [12], dynamic programming (DP) [13,14], two-stage stochastic programming [15], and distributionally stable simulation [16]. Multi-objective optimization techniques can be used as well as explained in [17]. Other optimization algorithms such as simulated annealing (SA) [18], teaching-learning-based optimization (TLBO) [19], backtracking search optimization (BSO) [20] and whales optimization algorithm (WOA) [21] could be used to solve optimization problems in power systems as well. One of the optimization problems is the optimal sizing problem of an ESS. One of the main approaches to address optimization problems under various uncertainties [22,23] is to use the chance-constrained process [24]. It is an optimization formulation that ensures the probability of reaching a certain constraint exceeds a certain amount. In other words, it limits the feasible region, so that the solution's confidence level is high. The chance-constrained method is a relatively robust approach but often hard to solve. In engineering [25] and finance [26], where uncertainties in price, demand, supply, currency exchange rate, recycling and feed rate, and demographic conditions are common, restricted opportunity optimization is particularly important. Some exemplary implementations of the chance-constrained approach include the management of water reserves and financial risk management. The method has been used more recently in unmanned autonomous vehicle navigation [27] and optimal renewable energy generation [24]. Other ESS optimization problems include the day-ahead scheduling [28], control algorithm [29], and demand flexibility [30]. Other techniques used to solve optimization problems in this area, such as chance-constrained information gap decision method [31] and robust optimization [32]. Moreover, the objective function does not have to minimize the total cost. It might maximize the use of renewable energy sources [33]. In this paper, the chance-constrained method [34] is used to solve the optimization problem and find the optimal size and location of an ESS and the optimal locations of feeders because the wind uncertainty is considered in the case study. Transmission expansion under uncertainty was discussed in [35].

Many power systems around the world are experiencing significant changes in generation portfolios. Environmental concern and energy stability, as well as rising fuel prices, have led to important, sustained growth in the capacity to generate wind and solar power worldwide. Variable generation, such as wind, solar , hydro and tidal, can be defined as those resources whose output depends on the conditions of the environment. The complexity of incorporating these variable resources into existing power systems varies depending on the variable resource size and scale, its connection with system load, and the versatility of the power system in question. Flexibility is here characterized as a system's ability to adapt its resources to respond to changes in net load, where net load is characterized as the remaining system load not represented by variable generation. Therefore, an isolated power system that mainly includes generation units with long start times and low ramp speeds would find it harder to incorporate variable generation successfully than a wellconnected power system, which contains several generation units that can quickly start and ramp. An emerging challenge in power system planning is to determine an existing system's ability to effectively implement targeted variable generation penetration, and thus to prepare potential portfolios. This paper will illustrate the need to assess flexibility in generation planning, analyze current planning practices and recommend an application of existing flexibility techniques to calculate flexibility [36]. 
Generation adequacy is a crucial quantification approach for evaluating supply security in an electrical system. The integration of high quantities of variable generation, demand response, storage, distributed generation, increased interconnection capacities, and coupling of the electricity markets motivates a need to review how adequacy is assessed. The indices included in this paper are loss of load probability (LOLP) and expected demand not supplied (EDNS). LOLP index shows the quality and performance of an electrical system. The growth influences the LOLP value in load, the load length curve, the plant's forced outage rate, the number, and the efficiency of the generating units. The EDNS measures the size of the load that has been lost due to the severe outage occurrence [37].

The aim of this paper is to develop a planning strategy to coordinate between integrating ESSs and capacity expansion of transmission lines and new tie-lines to accommodate the high integration of renewable power generation plants. The knowledge deficiencies in the literature are that there are no current frameworks to determine the scale and position of an ESS along with the optimum location of feeders for an efficient planning approach. The aim of this paper is to suggest an effective strategy to meet the needs of the next century with limited expenditure in the network infrastructure using ESS. The goal is to reduce the overall cost of acquisition and activities. The overall expense covers the expense of producing distributed turbines, the cost of building in ESS, and transmission line maintenance costs. Optimal design choices require the optimal sizes of ESSs, optimal distribution of size ESSs, and optimal combinations of expansion and/or additional transmission lines being planned. This paper further measures the feasibility of the suggested technique by measuring the LOLP and EDNS indices to assess how consistency of the approach strengthens and strengthens it. This article extends the approach proposed to a case study to illustrate the feasibility of the procedure proposed.

To summarize, the contributions of this paper are:

- Using the chance-constrained approach to reflect the uncertainty associated with the wind speed uncertainty.

- Identifying the optimal ESS sizes and positions.

- Identifying optimal tie-line options.

The advantages of this proposed methodology are:

- Co-optimization of the design approach to determine the optimum size and position of the ESS along with the optimal location of the feeders to increase the flexibility of the networks.

- Taking into account uncertainties about wind levels.

- The approach suggested provides greater flexibility as opposed to other approaches.

The drawback of the suggested approach is that in working with a huge number of scenarios, it is not a time-efficient technique. It is effective however, and does not require time to cope with a small range of scenarios.

The remainder of this paper is described as follows. Section 2 describes the proposed methodology to find the optimal ESS size and transmission line. Section 4 describes the case that will be studied to examine the proposed methodology. Section 5 illustrates the results and discusses them. Section 6 concludes the paper.

\section{Problem Formulation}

The problem formulation is subdivided into the following subgroups. Some equations in this section have been taken from [38].

\subsection{Objective Function}

The objective of the proposed methodology is to minimize the total cost. This is formulated in (1)-(5).

$$
C_{\text {Total }}=C_{G E N}+C_{E S S}+C_{T L}+C_{L O S S}
$$

where 
- $\quad C_{\text {Total }}$ is the total cost which should be minimized.

- $C_{G E N}$ is the generation cost related to distributed generators.

- $C_{E S S}$ is the investment cost related to ESS.

- $C_{T L}$ is the investment cost related to new transmission lines.

- $C_{\text {LOSS }}$ is the cost or penalties related to load shedding and wind curtailment.

$$
C_{G E N}=\sum_{g, t} a_{g} P_{g, t}^{2}+b_{g} P_{g, t}+c_{g}
$$

where $a_{g}, b_{g}$, and $c_{g}$ are the parameters of the cost functions of generators, and $P_{g, t}$ is the output power of generator $g$ at hour $t$.

$$
C_{E S S}=C_{P}^{E S S} \times P_{R}^{E S S}+C_{E}^{E S S} \times E_{R}^{E S S}
$$

where $C_{P}^{E S S}$ is the cost of power unit of the ESS, $P_{R}^{E S S}$ is the optimal power size of the ESS, $C_{E}^{E S S}$ is the cost of energy unit of the ESS, and $E_{R}^{E S S}$ is the optimal energy size of the ESS. The difference between the optimal power size and optimal energy size is that the power size is the rated power of the ESS. It is like the rated power of a generator when it is given in a power unit. On the other hand, the energy size is the capacity of the stored energy. It is like the capacity of a battery when it is given in an energy unit.

$$
C_{T L}=\left(-\eta_{i j}^{0}+\sum_{k, i j} \alpha_{i j}^{k}\right) C_{i j}
$$

where $e t a_{i j}^{0}$ is the initial status of branch connecting bus $i$ to bus $j$, this variable is binary, this means if there is a branch connecting bus $i$ to bus $j$, the value of this variable will be 1 , otherwise, it will be $0 . \alpha_{i j}^{k}$ is the investment decision of branch $k$ from bus $i$ to bus $j$, this variable is a binary variable as well and its value determines if the investment is feasible or not; the investment is feasible if its value is 1 , the variable $k$ indicates that there might be more than one line connecting bus $i$ to bus $j . C_{i j}$ is the investment cost of branch connecting bus $i$ to bus $j$. The minus sign in this equation is to exclude the current line from the cost calculation.

$$
C_{\text {LOSS }}=\sum_{i, t} V O L L \times P_{i, t}^{L S}+V W C \times P_{i, t}^{W C}
$$

where VOLL is the value of loss of load, $P_{i, t}^{L S}$ is the load shedding at bus $i$ at hour $t, V W C$ is the value of wind curtailment, and $P_{i, t}^{W C}$ is the wind curtailment at bus $i$ at hour $t$.

\subsection{Generator Constraints}

The generator constraints include its operational limitations, such as generation limits and ramp limits. These constraints are formulated in (6)-(8).

$$
P_{g}^{\min } \leq P_{g, t} \leq P_{g}^{\max }
$$

where $P_{g}^{\min }$ is the minimum limit of generator $g$, and $P_{g}^{\max }$ is the maximum limit of generator $g$.

$$
P_{g, t}-P_{g, t-1} \leq R U_{g}
$$

where $R U_{g}$ is the ramp up limit of generator $g$.

$$
P_{g, t-1}-P_{g, t} \leq R D_{g}
$$

where $R D_{g}$ is the ramp down limit of generator $g$. 


\subsection{ESS Constraints}

The ESS constraints are formulated in (9)-(14).

$$
P_{i, t}^{S}=P_{i, t}^{D}-P_{i, t}^{C}
$$

where $P_{i, t}^{S}$ is the output power of the ESS at bus $i$ at hour $t, P^{D} i, t$ is the discharging power of the ESS at bus $i$ at hour $t, P_{i, t}^{C}$ is the charging power of the ESS at bus $i$ at hour $t$.

$$
-P_{i}^{E S S} \leq P_{i, t}^{S} \leq P_{i}^{E S S}
$$

where $P_{i}^{E S S}$ is the power size of the ESS at bus $i$.

$$
\begin{gathered}
\sum_{i} P_{i}^{E S S}=P_{R}^{E S S} \\
E_{i, t}^{E}=E_{i, t-1}^{S}-P_{i, t}^{S} \Delta_{t}
\end{gathered}
$$

where $E_{i, t}^{S}$ is the stored energy in the ESS at bus $i$ at hour $t$, and $\Delta_{t}$ is the time step.

$$
0 \leq E_{i, t}^{S} \leq E_{i}^{E S S}
$$

where $E_{i}^{E S S}$ is energy size of the ESS at bus $i$.

$$
\sum_{i} E_{i}^{E S S}=E_{R}^{E S S}
$$

This model assumes that the ESS charges and discharges simultaneously, such as mobile batteries.

\subsection{Power Flow Constraints}

The constraints related to power flowing from a certain bus to another one are formulated in (15) and (16).

$$
\sum_{g, t} P_{g, t}+\sum_{i, t} P_{i, t}^{W}+P_{i, t}^{L S}+P_{i, t}^{S}-P_{i, t}^{L}=\sum_{i j} P_{i j, t}
$$

where $P_{i, t}^{W}$ is the wind power at bus $i$ at hour $t, P_{i, t}^{L}$ is the load at bus $i$ at hour $t$, and $P_{i j, t}$ is the power flow from bus $i$ to bus $j$ at hour $t$.

$$
P_{i j, t}=\frac{\delta_{i, t}-\delta_{j, t}}{x_{i j}}
$$

where $\delta_{i, t}$ is the voltage angle at bus $i$ at hour $t, \delta_{j, t}$ is the voltage angle at bus $j$ at hour $t$, and $x_{i j}$ is the reactance of the branch connecting bus $i$ to bus $j$.

\subsection{Power Loss Constraints}

There are two types of losses in this proposed methodology. These losses are either load shedding or wind curtailment. These losses are formulated in (17)-(19).

$$
\begin{gathered}
0 \leq P_{i, t}^{L S} \leq P_{i, t}^{L} \\
P_{i, t}^{W C}=W_{t} \Lambda_{i}^{\omega}-P_{i, t}^{W}
\end{gathered}
$$

where $W_{t}$ is the normalized wind power at hour $t$, and $\Lambda_{i}^{\omega}$ is the capacity of wind farm at bus $i$.

$$
0 \leq P_{i, t}^{W} \leq W_{t} \Lambda_{i}^{\omega}
$$




\subsection{Transmission Constraints}

The transmission constraints are formulated in (20)-(27).

$$
P_{i j, t}^{k}-B_{i j}\left(\delta_{i, t}-\delta_{j, t}\right) \leq\left(1-\alpha_{i j}^{k}\right) M
$$

where $P_{i j, t}^{k}$ is the power flow in branch $k$ from bus $i$ to bus $j$ at hour $t, B_{i j}$ is the susceptance of the branch connecting bus $i$ to bus $j$, and $M$ is the big number.

$$
\begin{aligned}
P_{i j, t}^{k} & -B_{i j}\left(\delta_{i, t}-\delta_{j, t}\right) \geq-\left(1-\alpha_{i j}^{k}\right) M \\
- & P_{i j}^{\max } \alpha_{i j}^{k} \leq P_{i j, t}^{k} \leq P_{i j}^{\max } \alpha_{i j}^{k}
\end{aligned}
$$

where $P_{i j}^{\max }$ is the maximum limit of branch connecting bus $i$ to bus $j$.

$$
\begin{aligned}
& \text { if } \eta_{i j}^{0}=1 \text { then } \alpha_{i j}^{k=1}=1 \\
& B_{i j}=\frac{1}{x_{i j}} \\
& \alpha_{i j}^{k} \in\{0,1\}
\end{aligned}
$$

and this indicates that $\alpha_{i j}^{k}$ is a binary variable.

$$
k \in\{1,2,3,4\}
$$

and this indicates that $k$ has four values. This implies that there is a possibility to add up to 4 branches between every two buses.

$$
M=\max _{i j} B_{i j}\left(\delta_{i}-\delta_{j}\right)
$$

\subsection{Chance-Constrained Method}

Chance-constrained method is a technique to solve stochastic optimization problems. This technique will be used in GAMS to solve the case study to show the results of the proposed methodology. A stochastic linear program can be expressed as follows:

$$
\begin{gathered}
\min _{x} C^{t} x \\
\text { subject to } P(A x \leq b) \geq \alpha \\
x \geq 0
\end{gathered}
$$

where $x$ is a decision variable, $c^{T}$ denotes the coefficients of the objective function, $A$ is a random matrix representing the coefficients, $b$ is a random vector denoting the right-hand side of the constraints, and $\alpha$ is the confidence level. The distinctive feature of chanceconstrained technique is that the constraints might be violated with the confidence level $\alpha$ where this confidence level is a value between 0 and 1 .

\subsection{Reliability Indices}

It is required to calculate the reliability indices to evaluate and assess the effectiveness of the proposed methodology and see if it gives better system reliability or not. In order to calculate LOLP, loss of energy expectation (LOEE) must be calculated first from the EDNS as formulated below.

$$
L O E E_{i}=\frac{\sum_{t} E D N S_{i, t}}{8736}
$$


where $L O E E_{i}$ is the loss of energy expectation at bus $i, E D N S_{i, t}$ is the expected demand not supplied at bus $i$ and hour $t, 8736$ is the number of hours in a year.

$$
L O L P_{i}=8736 * L O E E_{i}
$$

where $L O L P_{i}$ is the loss of load probability at bus $i$ per year.

\section{Proposed Solution Methodology}

There are many ways to solve a stochastic problem using the chance-constrained technique. One of these ways is to convert the formulation to a deterministic mixedinteger problem. The idea here is to introduce and define a set of multiple scenarios and a vector with binary variables for each scenario. The binary variables must be 1 when the corresponding constrained is satisfied in a certain scenario, otherwise, the binary variable must be 0 . This chance-constrained formulation which is based on scenarios can be written as formulated in (33)-(36).

$$
\begin{gathered}
\min _{x} C^{T} x \\
\text { subject to } A^{k} \leq b^{k}+M^{k}\left(1-y_{k}\right) \\
\sum_{k \in S} y_{k} \geq \alpha \times|S| \\
x \geq 0, y \in(0,1)^{|S|}
\end{gathered}
$$

where $k$ is a scenario index, $S$ is a set of scenarios, $y_{k}$ is a binary variable, $M_{k}$ is a big-M vector that should be chosen to avoid cutting off any feasible solution if the corresponding constraint is not satisfied, which means $y_{k}=0$.

In simple cases, where decision and random variables can be decoupled and the constraint can be relaxed by using probability density functions in deterministic constraints, and standard linear programming or non-linear programming methods can be used to solve the problem. However, the transformation of chance constraints into deterministic constraints, mainly linked to structure, convexity and stability, poses many challenges. In more complex cases where decision and random variables will interact in such a way as to make it difficult to decouple them, the problem cannot be solved at present.

A problem is called linear if it is possible to use linear transformation of multivariate distributed variables to estimate the constraints distribution. The method to address such an issue is to simplify the issue into analogous deterministic issues. In other words, one can calculate the probability using the function of probability density and replace a deterministic expression on the left-hand side of the constraint. Usually, if ample data is available, these functions of probability density can be obtained by linear regression, or interpolation and extrapolation if not much data is accessible. In cases where uncertain variables have correlations, the probability can be calculated using marginal distribution functions. The feasible region of luck-constrained issues depends on the level of confidence desired by the user. If, as in cases dealing with safety parameters, a high level of confidence is required, the feasible region is small. By reducing the confidence level, the feasible area becomes larger. In certain cases where one constraint is more important than another, the problem can be formulated in such a way that the trust level of one constraint is higher for the greater constraint.

Due to uncertainties in demand, supply, quality , energy availability etc., the chanceconstraint approach is most frequently used in engineering as well as finance. The chanceconstraint approach has historically been very useful in the control of power systems. Energy availability has brought in uncertainties. Sustainable energy such as wind and solar energy contained many uncertainties since wind velocity varies significantly throughout the year and solar intensity can vary depending on the time of day or location. To ensure that a power plant can satisfy energy demand at least to a certain degree of confidence, the chance-constraint approach has been formulated in [28-30] can be used. The limitations 
of the proposed methodology are that they are not perfect for large systems which require composition techniques to solve their optimization problems.

\section{Case Study}

The IEEE Reliability Test System-1996 (RTS-96) is considered to be a case study for optimal planning of an ESS and transmission line [39]. The RTS-96 has 12 distributed generators which are distributed among 24 buses. The RTS-96 does not have renewable energy sources and it has a certain number of transmission lines as described in [39]. This system will be modified by adding wind farms in this paper. It is required to optimally size and allocate ESSs in the system. In addition, new transmission lines, feeders, will be added in their optimal selections. The difference between the original RTS-96 system and the system after the integration of ESSs and feeders is that the new system will be more economic and reliable. The objective is to apply the proposed methodology in this paper to find the total optimal sizes of ESSs to be added to this system. Then, it is required to allocate among the system buses. Simultaneously, if there is a need to reinforce or add a new transmission line, it will be suggested. The load profile has been taken from the RTS-96 for a one-year horizon. Figure 1 shows the load curve of the whole horizon.

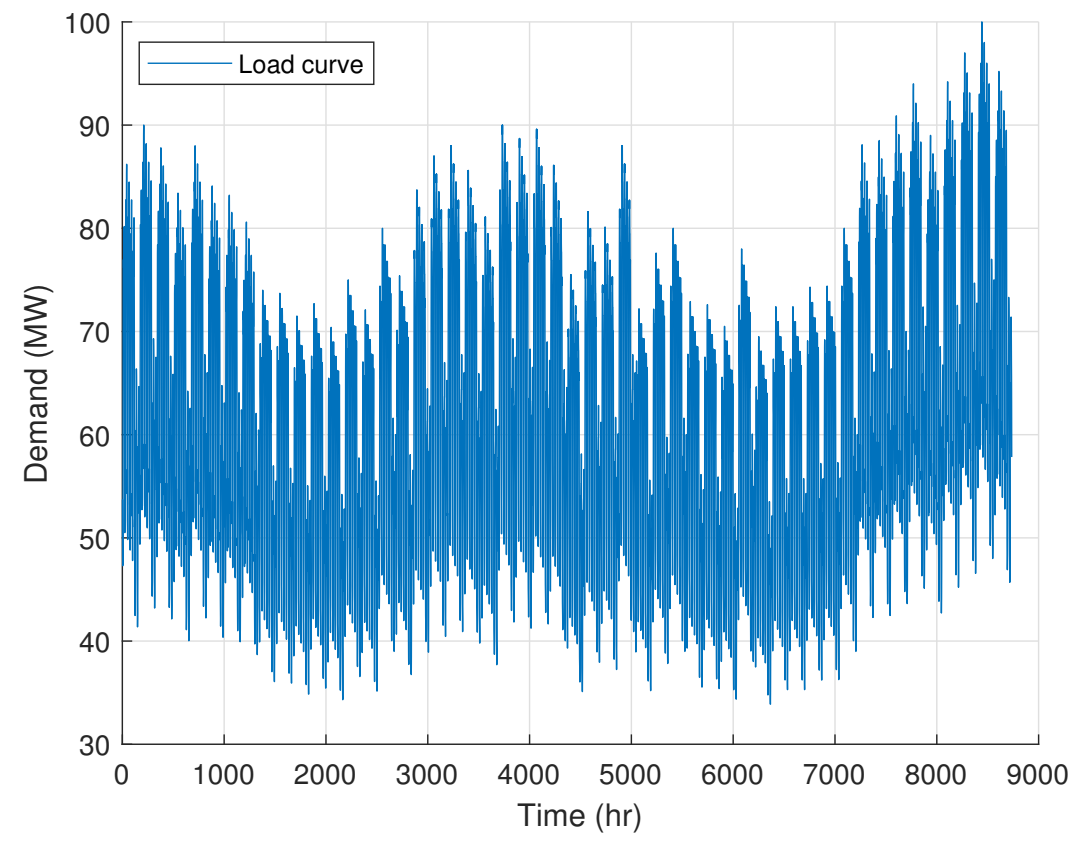

Figure 1. Load curve illustrating the demand in MW at every hour during the year.

Figure 2 shows the load duration curve of the load profile. It presents the minimum load demand during any itemized time slot. It authorizes the selection of base-load and peak-load needed from the power system operator to manage for the generation. Any spot on the curve describes the total span in hours for the corresponding load demand. 


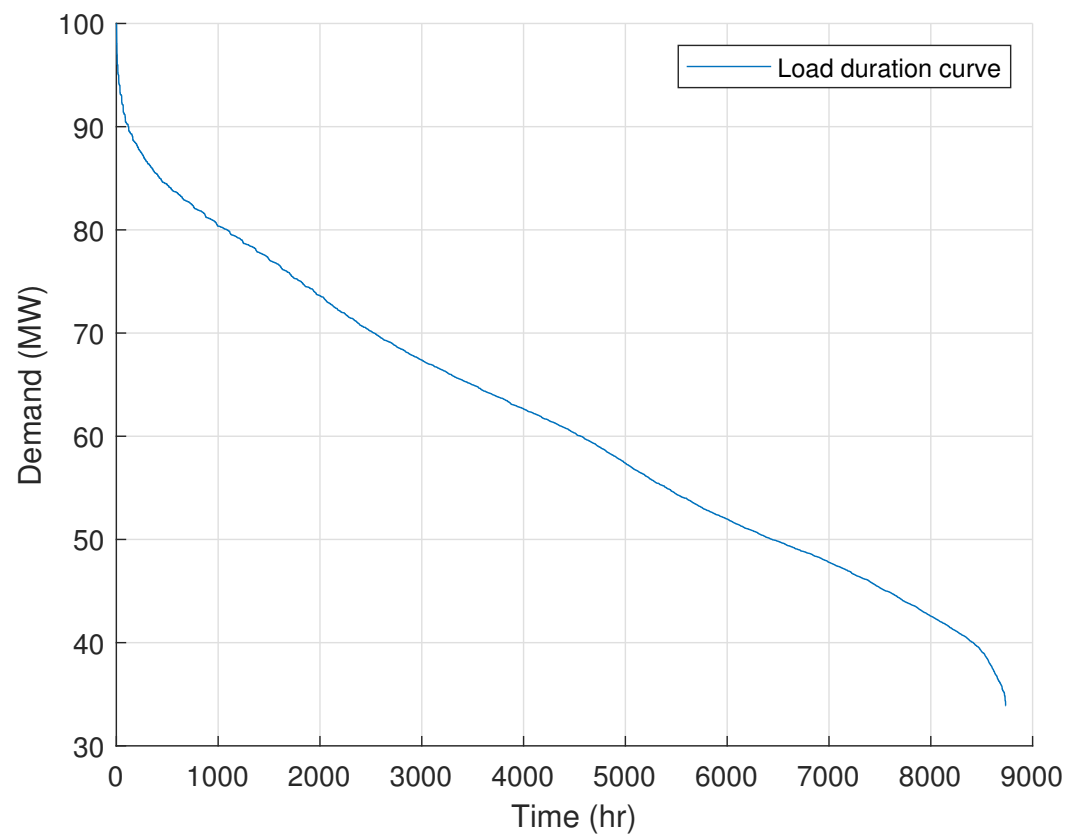

Figure 2. Load duration curve.

Wind farms have been added to the RTS-96 and connected to specific buses. Moreover, Table 1 shows the capacities of wind farms at those buses which are 8, 19, and 21 . The normalized wind power curve is used to calculate the output power of each wind farm.

Table 1. Capacities of wind farms at the buses.

\begin{tabular}{cccc}
\hline Bus & Bus 8 & Bus 19 & Bus 21 \\
\hline Capacity & $200 \mathrm{MW}$ & $150 \mathrm{MW}$ & $100 \mathrm{MW}$ \\
\hline
\end{tabular}

In the case study, to assess the effectiveness of the proposed methodology, the results of the deterministic method and the results of the probabilistic method, which is the proposed methodology, will be compared with the results of the base case in terms of $C_{T}$, LOLP, EDNS, system availability and unavailability. The base case is the system without the ESS and new feeders. This means the system before solving the optimization problem will be compared to the system after solving the problem using two methods. After that, the results of both methods will be compared with each other to highlight the improvement in the results of the proposed methodology. The values of parameters used in the case study are illustrated in Table 2. Other parameters are taken from the RTS-96.

Table 2. Values of parameters used in the case study.

\begin{tabular}{cc}
\hline Parameter & Value \\
\hline$\Delta_{t}$ & $1 \mathrm{~h}$ \\
\hline$C_{P}^{E S S}$ & $\$ 120 / \mathrm{MW}$ \\
\hline$C_{E}^{E S S}$ & $\$ 30 / \mathrm{MWh}$ \\
\hline$V O L L$ & $\$ 10,000$ \\
\hline$V W C$ & $\$ 50$ \\
\hline
\end{tabular}




\section{Results and Discussions}

The stochastic optimal planning problem has been tested using chance-constrained method to fulfill the minimum investment and operational cost, which is $\$ 83,940$. The aggregated optimal size of the ESSs is $87.7 \mathrm{MW}$ and $163.2 \mathrm{MWh}$. The ESSs have been optimally allocated and distributed to Buses 8, 16, 17, and 22. Table 3 illustrates how the ESS is distributed among those buses. The results in Table 3 are illustrated in Figure 3. It can be noted that the ESSs have been allocated to four buses. Two of them are load buses, which are Buses 8 and 16. Also, the other two buses, which are Buses 17 and 22, do not have load points. Bus 22 has the largest ESS in this system while Bus 16 has the smallest ESS. Regarding the transmission expansion planning, it is recommended to add a feeder from Bus 19 to Bus 21 and another feeder from Bus 20 to Bus 22. Figure 4 shows the singleline diagram of the RTS-96 with the new transmission lines represented as dashed lines. These two feeders will contribute significantly to minimizing the total cost. Regarding losses, there is no load shedding in this case study. However, there is a wind curtailment of 27.6 MWh. Figure 5 illustrates the different solutions of different confidence levels using the chance-constrained technique and. In addition, Figure 4 compares these different solutions with solution resulted from the deterministic optimization to highlight the effectiveness of the chance-constrained technique. As shown in this figure, when the confidence level decreases, the optimality of the solution decreases as well. Therefore, the confidence level should be kept as high as possible to maintain high optimality and flexibility.

To prove the effectiveness of the proposed methodology, the total cost without investing in the ESS and transmission lines is $\$ 92,530$. This indicates that the total cost has decreased by $9.28 \%$ in a one-year plan. The results could be improved more for longer horizons.

The insufficient ramping resource expectation (IRRE) is the estimated number of observations when a power system is unable to cope, predicted or unpredicted, with changes in net charge. Calculation of the IRRE follows a similar structure to the LOLE, but instead of forming a distribution of the inaccessible generation power, a distribution of the available versatile resources for each direction and time horizon is created. Second, as with the LOLE calculation, the probability that at each observation the device has insufficient ramp resources, over each time horizon and path, is calculated from the available flexibility distribution), (from which the overall metrics were calculated. Calculating the Value over all chosen time horizons offers an understanding of the capacity of the resources of a device to fulfill the uncertainty requirements of the net price. The IRRE is calculable in a series of measures [36]. The following steps are followed to calculate the IRRE. Figure 6 illustrates the upward flexibility of this system in the basic, deterministic, and probabilistic cases to highlight the high flexibility after integrating the ESS. In addition, Figure 7 illustrates the down flexibility of the system in the three cases.

Table 3. ESS allocation by power and energy.

\begin{tabular}{ccccc}
\hline Bus & Bus 8 & Bus 16 & Bus 17 & Bus 22 \\
\hline Power (MW) & 27.7 & 3.5 & 14.0 & 42.5 \\
Energy (MWh) & 50.3 & 8.4 & 19.8 & 84.7 \\
\hline
\end{tabular}

- Calculation of net load ramping time series in both upward and downward (dn) directions for the entire planning horizon.

- Calculation of the versatile resources available up/dn within a defined time frame of interest (e.g., one hour), provided the availability and engagement status of each generation unit, start-up time, actual output level and total up- or down-ramping capabilities for the next cycle. 
- To combine all time series to achieve the complete up/dn versatility time series available for all tools.

- Calculation of the empirical cumulative distribution function of up/dn available from the complete versatility time series available.

- Calculating the likelihood of inadequate ramping by removing the net load ramping needed in the obtained distribution function. The total of the time series up/down probabilities gives the IRRE.

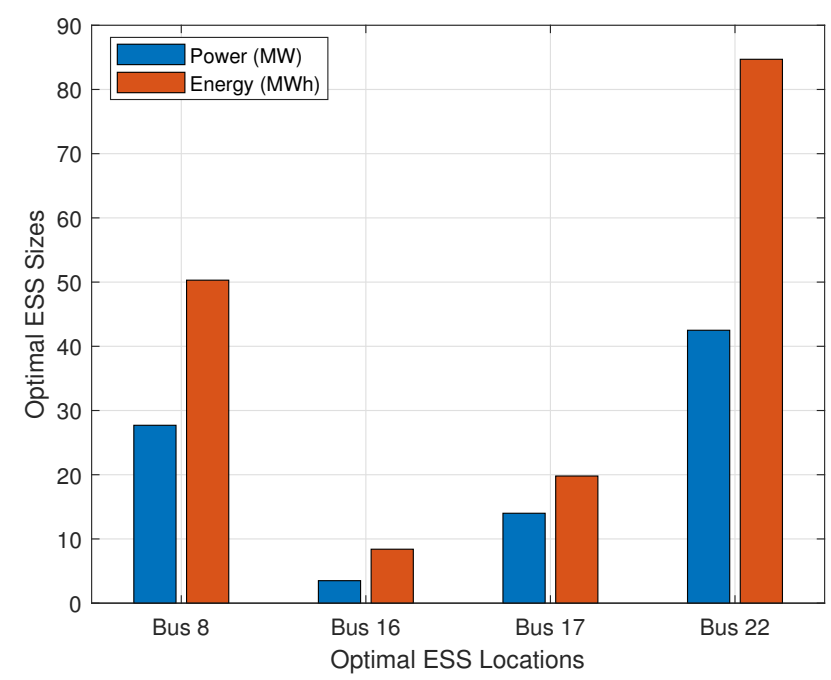

Figure 3. Optimal ESS sizes and locations.



Figure 4. Single-line diagram of the RTS-96 with new feeders. 


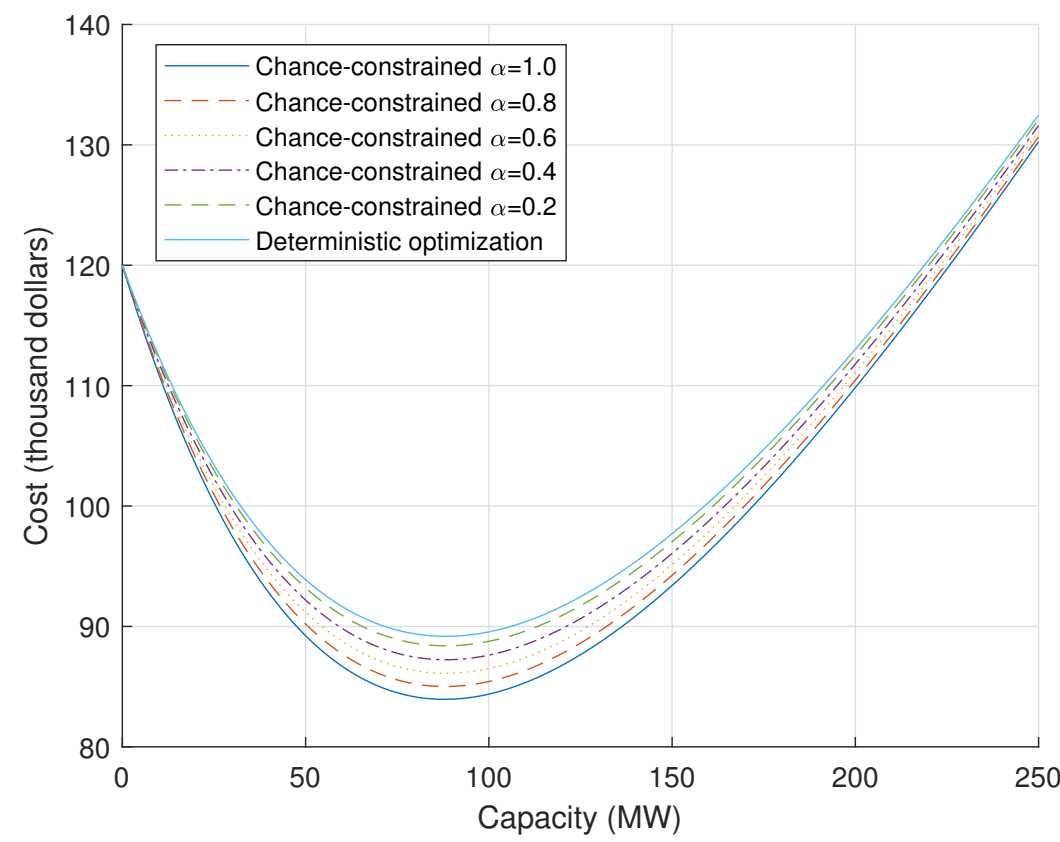

Figure 5. Cost comparison of different confidence levels.

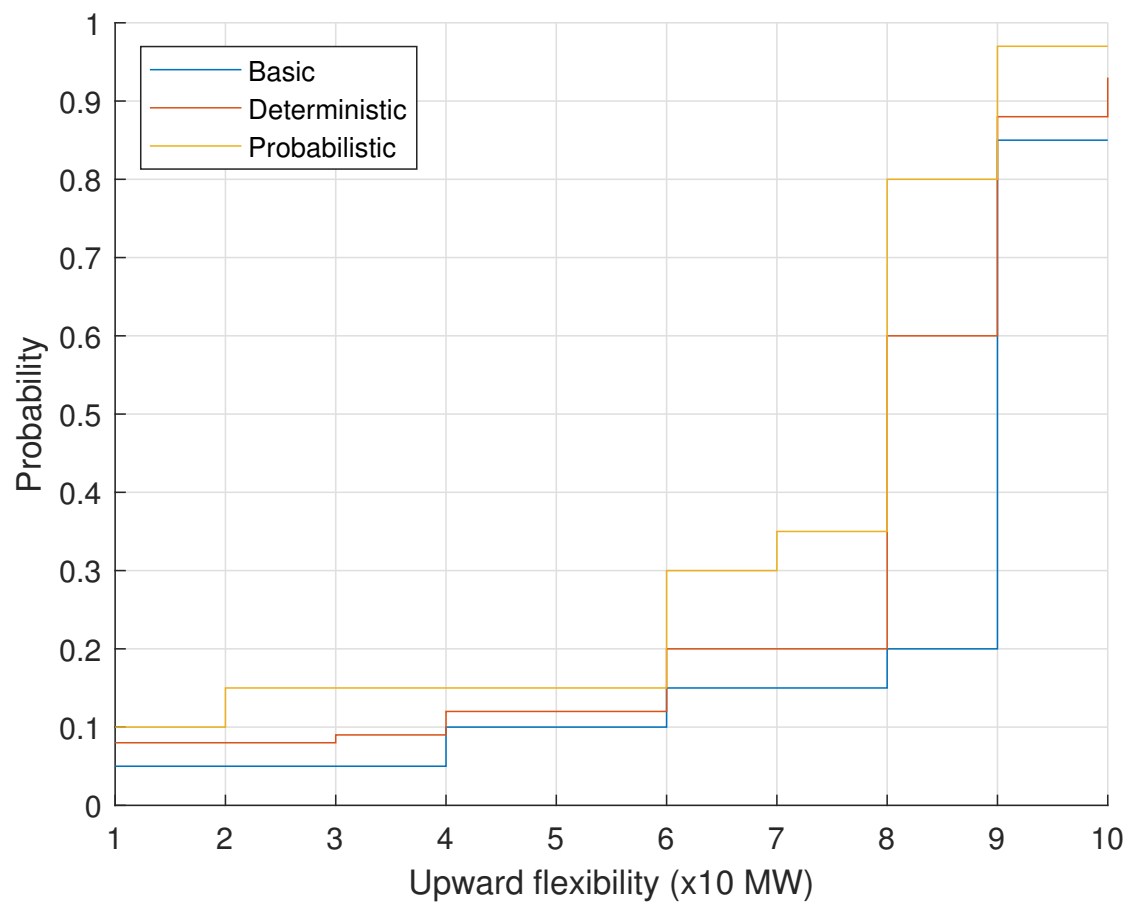

Figure 6. Upward flexibility. 


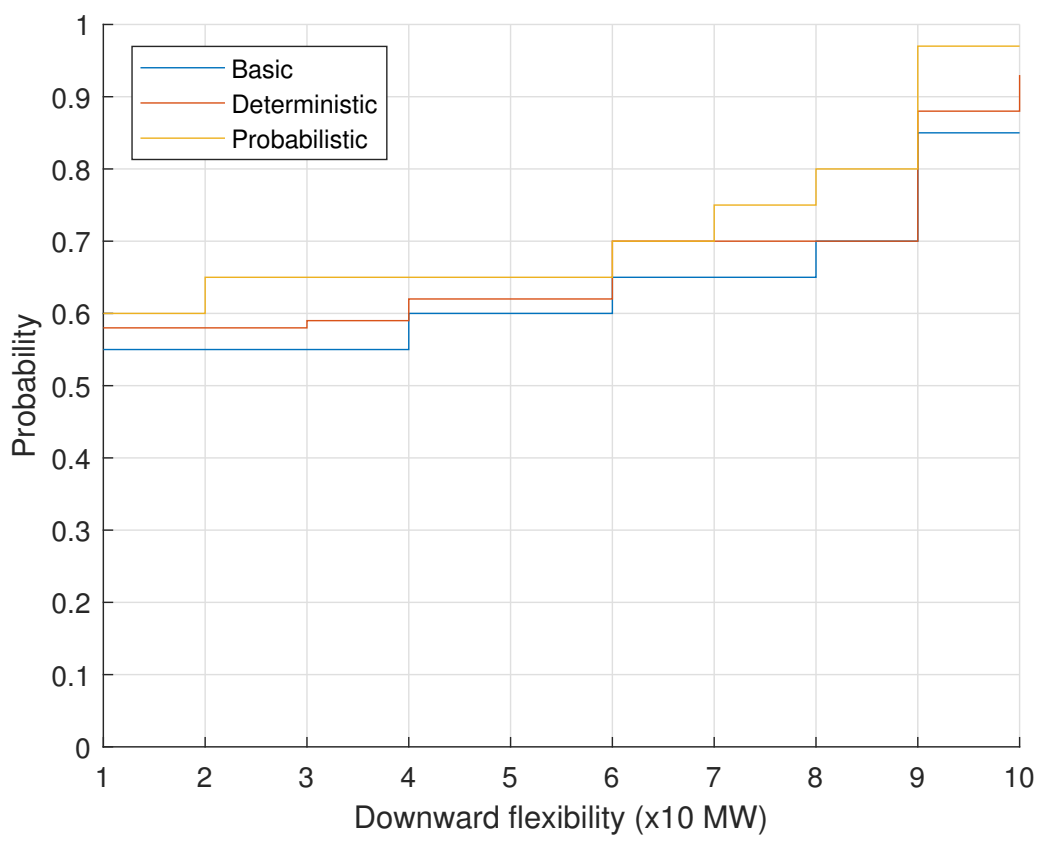

Figure 7. Downward flexibility.

In addition, the generation adequacy has been assessed in order to highlight the effectiveness of the proposed methodology in comparison with the base case. The LOLP and EDNS indices have been calculated in the base case, deterministic case, and probabilistic case which has been solved using the chance-constrained method. Average System Availability Index (ASAI) and Average System Unavailability Index (ASUI) have been included as well. Table 4 summarizes this data. Table 5 shows the improvement of all terms of both deterministic and probabilistic cases in reference and comparison to the base case. As shown in Table 5, the probabilistic method, which is the chance-constrained technique, gave better improvement than the deterministic method. These results in Tables 4 and 5 are visualized in Figures 8 and 9, respectively. As illustrated in Figure 9, the minimum cost, LOLP, EDNS, and ASUI are in the probabilistic case. This means that this method has the most optimal results among all cases. In addition, Figure 8 illustrates that the probabilistic method has better effectiveness, improvement, and enhancement compared with the deterministic case.

Table 4. Comparison between the three cases.

\begin{tabular}{cccc}
\hline Term & Base & Deterministic & Probablistic \\
\hline$C_{T}$ & $\$ 92,530$ & $\$ 89,210$ & $\$ 83,940$ \\
$L O L P$ & $78.23 \%$ & $71.18 \%$ & $66.47 \%$ \\
$E D N S$ & $13.14 \mathrm{MWh}$ & $11.36 \mathrm{MWh}$ & $10.21 \mathrm{MWh}$ \\
Availability & $97.9995 \%$ & $98.9395 \%$ & $99.9914 \%$ \\
Unavailability & $2.0005 \%$ & $1.0605 \%$ & $0.0086 \%$ \\
\hline
\end{tabular}

Table 5. Effectiveness of proposed methodology compared to the base case.

\begin{tabular}{ccc}
\hline Term & Deterministic & Probabilistic \\
\hline$C_{T}$ & $3.59 \%$ & $9.28 \%$ \\
$L O L P$ & $9.01 \%$ & $15.03 \%$ \\
$E D N S$ & $13.55 \%$ & $22.30 \%$ \\
Availability & $0.96 \%$ & $2.03 \%$ \\
\hline
\end{tabular}


The model was developed using the linear mixed-integer programming to reflect the charging/discharging essence of the ESS operation. The power balance restriction was established using DC-OPF, which is suitable for the planning studies at the transmission systems level. The proposed planning strategy was evaluated using the IEEE RTS-96 test system. The planning decisions were found to serve the projected load demand and consider the new integrated wind power plants. The proposed approach was considered with various confidence levels to demonstrate the variations in the planning decisions as the confidence level is adjusted. The generation adequacy measures in terms of LOLP and EDNS were quantified to illustrate the approach's performance. Flexibility is assessed by measuring the upward and down wared ramping capabilities.

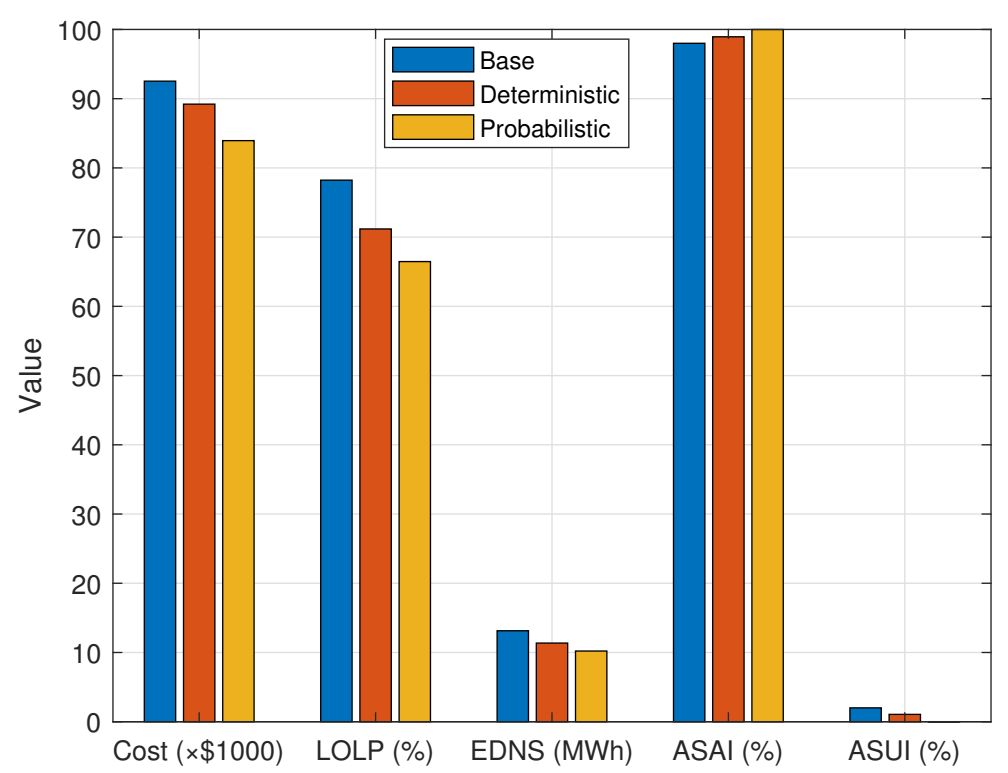

Figure 8. Visualized comparison of the three cases.



Figure 9. Visualized effectiveness of proposed methodology compared to the base case. 


\section{Conclusions}

This paper proposed a chance-constraint robust co-optimization strategy to find the optimum sizes and locations of the ESSs combined with transmission lines planning to accommodate the high integration of wind power generation plants under uncertainties. The approach provided the the optimum range of the required new tie-lines or excited ones needed to be reinforced to maximize the use of variable wind power resources. As a continuation of this paper, wind power uncertainty representation could be investigated using a different approach such as Distributionally Robust Optimization. By considering additional system components such as solar power generation plants, various ESS technologies measure the contributions of distributions systems. The proposed methodology is quite general and straightforward, easily extended to power grid modernization such as future smart grids. The contributions of this paper are using the chance-constrained approach to reflect the uncertainty associated with the wind speed uncertainty, identifying the optimal ESS sizes and positions, and identifying optimal tie-line options. The results of the proposed methodology can be used to integrate an ESS with a certain power system and add new feeders for the transmission expansion planning. This will help in reducing the costs and enhancing the system reliability. The investment cost of the ESS and feeders could be high, but the total cost of the investment and operation will be less than the operation cost of the original system for the long-term planning.

Author Contributions: Conceptualization, F.A., M.A.A. and M.K.; Data curation, M.A.A.; Formal analysis, F.A. and M.A.A.; Funding acquisition, F.A. and M.K.; Investigation, F.A. and M.A.A.; Methodology, F.A. and M.A.A.; Project administration, F.A. and M.K.; Resources, F.A., M.A.A. and M.K.; Software, F.A. and M.A.A.; Supervision, F.A. and M.K.; Validation, F.A., M.A.A. and M.K.; Visualization, F.A. and M.A.A.; Writing—original draft, F.A.; Writing—review \& editing, F.A., M.A.A. and M.K. All authors have read and agreed to the published version of the manuscript.

Funding: The authors would like to acknowledge the support provided by the Deanship of Research (DSR) at King Fahd University of Petroleum \& Minerals (KFUPM) for funding this work through project No. DF181035. In addition, the authors would like to acknowledge the funding support provided by the King Abdullah City for Atomic and Renewable Energy (K.A.CARE).

Data Availability Statement: Not applicable.

Conflicts of Interest: The funders had no role in the design of the study; in the collection, analyses, or interpretation of data; in the writing of the manuscript, or in the decision to publish the results.

\section{Abbreviations}

The following abbreviations are used in this manuscript:

$\begin{array}{ll}\alpha_{i j}^{k} & \text { Investment decision of branch } k \text { from bus } j \\ \Delta_{t} & \text { Time step } \\ \delta_{i, t} & \text { Voltage angle at bus } i \text { at hour } t \\ \eta_{i j}^{0} & \text { Initial status of branch connecting bus } i \text { to bus } j \\ \Lambda_{i}^{\omega} & \text { Capacity of wind farm at bus } i \\ P_{i, t}^{W} & \text { Uncertain wind data } \\ a_{g}, b_{g}, c_{g} & \text { Parameters of cost functions of generators } \\ B_{i j} & \text { Susceptance of branch connecting bus } i \text { to bus } j \\ C_{E}^{E S S} & \text { Cost of energy unit of ESS } \\ C_{P}^{E S S} & \text { Cost of power unit of ESS } \\ C_{E S S} & \text { Investment cost related to ESS } \\ C_{G E N} & \text { Generation cost related to distributed generators } \\ C_{i j} & \text { Investment cost of branch connecting bus } i \text { to bus } j \\ C_{L O S S} & \text { Cost or penalties related to load shedding and wind curtailment } \\ C_{T L} & \text { Investment cost related to new transmission lines } \\ C_{T o t a l} & \text { Total cost } \\ E_{i}^{E S S} & \text { Energy size of ESS at bus } i\end{array}$






\section{References}

1. Bahramirad, S.; Camm, E. Practical modeling of Smart Grid SMSTM storage management system in a microgrid. In Proceedings of the IEEE Power Engineering Society Transmission and Distribution Conference, Orlando, FA, USA, 7-10 May 2012; pp. 1-7.

2. Adefarati, T.; Bansal, R.C. Integration of renewable distributed generators into the distribution system: A review. IET Renew. Power Gener. 2016, 10, 873-884. [CrossRef]

3. Bahramirad, S.; Reder, W.; Khodaei, A. Reliability-constrained optimal sizing of energy storage system in a microgrid. IEEE Trans. Smart Grid 2012, 3, 2056-2062. [CrossRef]

4. Chen, S.X.; Gooi, H.B.; Wang, M.Q. Sizing of energy storage for microgrids. IEEE Trans. Smart Grid 2012, 3, 142-151. [CrossRef]

5. Gao, D.W. Energy Storage for Sustainable Microgrid; Academic Press: Cambridge, MA, USA, 2015.

6. Singh, S.; Singh, M.; Kaushik, S.C. Optimal power scheduling of renewable energy systems in microgrids using distributed energy storage system. IET Renew. Power Gener. 2016, 10, 1328-1339. [CrossRef]

7. Upendra Roy, B.P.; Rengarajan, N. Feasibility Study of an Energy Storage System for Distributed Generation System in Islanding Mode. J. Energy Resour. Technol. Trans. ASME 2017, 139. [CrossRef]

8. Elsir, M.T.; Abdulgalil, M.A.; Al-Awami, A.T.; Khalid, M. Sizing and allocation for solar energy storage system considering the cost optimization. In Proceedings of the 8th International Conference on Renewable Energy Research and Applications, ICRERA 2019, Brasov, Romania, 3-6 November 2019; pp. 407-412.

9. Abdulgalil, M.A.; Khalid, M. Enhancing the reliability of a microgrid through optimal size of battery energy storage system. IET Gener. Transm. Distrib. 2019, 13, 1499-1508. [CrossRef]

10. Bahramirad, S.; Daneshi, H. Optimal sizing of smart grid storage management system in a microgrid. In Proceedings of the 2012 IEEE PES Innovative Smart Grid Technologies, ISGT 2012, Washington, DC, USA, 16-20 January 2012; pp. 1-7.

11. Salman, U.T.; Abdulgalil, M.A.; Wasiu, O.S.; Khalid, M. Energy management strategy considering battery efficiency for grid-tied microgrids during summer in the Kingdom of Saudi Arabia. In Proceedings of the 8th International Conference on Renewable Energy Research and Applications, ICRERA 2019, Brasov, Romania, 3-6 November 2019; pp. 422-427.

12. Gupta, P.P.; Jain, P.; Sharma, S.; Bhakar, R. Reliability-Security Constrained Unit Commitment based on benders decomposition and Mixed Integer Non-Linear Programming. In Proceedings of the 2017 International Conference on Computer, Communications and Electronics, Comptelix 2017, Sanya, China, 25-26 June 2017; pp 328-333.

13. Nguyen, T.A.; Crow, M.L.; Elmore, A.C. Optimal sizing of a vanadium redox battery system for microgrid systems. IEEE Trans. Sustain. Energy 2015, 3, 729-737. [CrossRef]

14. Abdulgalil, M.A.; Khater, M.N.; Khalid, M.; Alisamail, F. Sizing of energy storage systems to enhance microgrid reliability. In Proceedings of the IEEE International Conference on Industrial Technology, Lyon, France, 19-22 February 2018; pp. 1302-1307. 
15. Huang, Y.; Pardalos, P.M.; Zheng, Q.P. Two-Stage Stochastic Programming Models and Algorithms; Springer: Berlin/Heidelberg, Germany, 2017.

16. Xiong, P.; Jirutitijaroen, P.; Singh, C. A Distributionally Robust Optimization Model for Unit Commitment Considering Uncertain Wind Power Generation. IEEE Trans. Power Syst. 2017, 32, 39-49. [CrossRef]

17. Kim, H.J.; Kim, M.K. Multi-Objective Based Optimal Energy Management of Grid-Connected Microgrid Considering Advanced Demand Response. Energies 2019, 12, 4142. [CrossRef]

18. Ma, Y.; Xie, K.; Dong, J.; Tai, H. M.; Hu, B. Optimal Generation Maintenance Schedule for Bundled Wind-Thermal Generation System. J. Energy Resour. Technol. Trans. ASME 2018, 140. [CrossRef]

19. Javad Kasaei, M.; Gandomkar, M.; Nikoukar, J. Optimal Operational Scheduling of Renewable Energy Sources Using TeachingLearning Based Optimization Algorithm by Virtual Power Plant. J. Energy Resour. Technol. Trans. ASME 2017, 139. [CrossRef]

20. Li, Y.; Mohammed, S.Q.; Nariman, G.S.; Aljojo, N.; Rezvani, A.; Dadfar, S. Energy management of microgrid considering renewable energy sources and electric vehicles using the backtracking search optimization algorithm. J. Energy Resour. Technol. Trans. ASME 2020, 142. [CrossRef]

21. Ma, S.; Zhou, D.; Zhang, H.; Weng, S.; Shao, T. Modeling and Operational Optimization Based on Energy Hubs for Complex Energy Networks with Distributed Energy Resources. J. Energy Resour. Technol. Trans. ASME 2019, 141. [CrossRef]

22. Abdulgalil, M.A.; Khalid, M.; Alismail, F. Optimizing a Distributed Wind-Storage System Under Critical Uncertainties Using Benders Decomposition. IEEE Access 2019, 7, 77951-77963. [CrossRef]

23. Abdulgalil, M.A.; Khalid, M.; Alismail, F. Optimal Sizing of Battery Energy Storage for a Grid-Connected Microgrid Subjected to Wind Uncertainties. Energies 2019, 12, 2412. [CrossRef]

24. Yahya Soltani, N.; Nasiri, A. Chance-Constrained Optimization of Energy Storage Capacity for Microgrids. IEEE Trans. Smart Grid 2020, 11, 2760-2770. [CrossRef]

25. Daneshvar, M.; Mohammadi Ivatloo, B.; Abapour, M.; Asadi, S.; Khanjani, R. Distributionally Robust Chance Constrained Transactive Energy Framework for Coupled Electrical and Gas Microgrids. IEEE Trans. Ind. Electron. 2020, 68, 347-357. [CrossRef]

26. Lee, Y.J.; Joo, S.J.; Hwang, T. An analysis of Korean bank performance using chance-constrained data envelopment analysis. Int. J. Oper. Res. 2020, 38, 525-543. [CrossRef]

27. 1. Castillo-Lopez, M.; Ludivig, P.; Sajadi-Alamdari, S. A.; Sanchez-Lopez, J. L.; Olivares-Mendez, M. A.; Voos, H. A Real-Time Approach for Chance-Constrained Motion Planning with Dynamic Obstacles. IEEE Robot. Autom. Lett. 2020, 5, 3620-3625. [CrossRef]

28. Silva, V.A.; Aoki, A.R.; Lambert-Torres, G. Optimal Day-Ahead Scheduling of Microgrids with Battery Energy Storage System. Energies 2020, 13, 5188. [CrossRef]

29. Adamczyk, A. Sizing and Control Algorithms of a Hybrid Energy Storage System Based on Fuel Cells. Energies 2020, 13, 5147. [CrossRef]

30. Fambri, G.; Badami, M.; Tsagkrasoulis, D.; Katsiki, V.; Giannakis, G.; Papanikolaou, A. Demand Flexibility Enabled by Virtual Energy Storage to Improve Renewable Energy Penetration. Energies 2020, 13, 5128. [CrossRef]

31. Wei, J.; Zhang, Y.; Wang, J.; Cao, X.; Khan, M.A. Multi-period planning of multi-energy microgrid with multi-type uncertainties using chance constrained information gap decision method. Appl. Energy 2020, 260, 114188. [CrossRef]

32. Chowdhury, N.; Pilo, F.; Pisano, G. Optimal energy storage system positioning and sizing with robust optimization. Energies 2020, 13, 512. [CrossRef]

33. Li, Q.; Wang, J.; Zhang, Y.; Fan, Y.; Bao, G.; Wang, X. Multi-period generation expansion planning for sustainable power systems to maximize the utilization of renewable energy sources. Sustainability 2020, 12, 1083. [CrossRef]

34. Ozturk, U.A.; Mazumdar, M.; Norman, B.A. A solution to the stochastic unit commitment problem using chance constrained programming. IEEE Trans. Power Syst. 2004, 19, 1589-1598. [CrossRef]

35. Kim, W.W.; Park, J.K.; Yoon, Y.T.; Kim, M.K. Transmission expansion planning under uncertainty for investment options with various lead-times. Energies 2018, 11, 2429. [CrossRef]

36. Lannoye, E.; Flynn, D.; O'Malley, M. Evaluation of power system flexibility. IEEE Trans. Power Syst. 2012, 27, 922-931. [CrossRef]

37. Alismail, F.; Xiong, P.; Singh, C. Optimal Wind Farm Allocation in Multi-Area Power Systems Using Distributionally Robust Optimization Approach. IEEE Trans. Power Syst. 2018, 33, 536-544. [CrossRef]

38. Soroudi, A. Power System Optimization Modeling in GAMS; Springer: Berlin/Heidelberg, Germany, 2017.

39. Grigg, C.; Wong, P. The IEEE reliability test system-1996 a report prepared by the reliability test system task force of the application of probability methods subcommittee. IEEE Trans. Power Syst. 1999, 14, 1010-1020. [CrossRef] 